hep-ph/9704265

\title{
Conformal symmetry on the light cone
}

\author{
and nonleading twist distribution amplitudes
}

\author{
of massive vector meson
}

\author{
Igor Halperin \\ Physics and Astronomy Department \\ University of British Columbia \\ 6224 Agriculture Road, Vancouver, BC V6T 1Z1, Canada \\ e-mail: higor@physics.ubc.ca
}

\begin{abstract}
:
A complete set of asymptotic three particle light cone distribution amplitudes of twist 3 and 4 for a transversely polarized massive vector meson built out of massless current quarks is constructed. The method used is based on a modified conformal projectors technique which allows to handle kinematical corrections due to a finite hadron mass. Consequences of our finding for the $\rho$-meson hard diffractive electroproduction and $\gamma \rho \pi$ form factor are discussed. Our results may imply a breakdown of OPE for some exclusive processes beyond the leading twist level.
\end{abstract}

submitted to Phys. Lett. B 


\section{Introduction}

Light cone distribution amplitudes (DA's) of hadrons are the key ingredient of the modern QCD approach to hard [1] and soft [2] exclusive, diffractive [3] and deep inelastic scattering 4 processes. They provide the universal non-perturbative input in physical amplitudes where the only dependence on a particular process enters as a normalization point of a corresponding DA. Respectively, they have to be addressed using non-perturbative methods. The approach of the QCD sum rules pioneered for the study of light cone DA's by Chernyak and Zhitnitsky more than a decade ago [5] hitherto remains the most popular and reliable among methods intended to handle this problem.

While a good deal of information was obtained on leading twist DA's for different hadrons, relatively a little has been known about nonleading twist DA's except for the case of pion. A systematic approach to the study of higher twist DA's was suggested in [6] where a complete set of light cone DA's of twist 3 and 4 for the pion has been constructed and studied in detail. The analysis of Ref. [6] was heavily based on group-theoretical methods exploiting the conformal symmetry which holds in QCD at the one-loop level [7, 8, 9]. The conformal invariance has proved useful for the study of leading twist DA's 10 where it allows to diagonalize the mixing matrix at one loop. The use of the conformal symmetry becomes even more crucial in the analysis of nonleading twist DA's where methods based on direct calculations of suitable correlation functions turn out either too complicated or erroneous [6].

The aim of this letter is to extent the conformal group methods of Ref.[6] to a study of nonleading twist three particle light cone DA's for a massive vector meson built out of massless current quarks . Fortunately, it requires only a slight modification of the technique developed in [6] to handle kinematical corrections due to a non-vanishing hadron mass. This method yields quite a different classification and new results for twist 4 DA's in comparison to the analysis of Ref.[0]. Our interest in this problem is mainly due to its utility for the diffractive vector meson electroproduction at HERA [12] (see [13 for updated review of the experimental and theoretical state of affair there). Recently the perturbative QCD approach of Ref. [3] to the diffractive electroproduction of a longitudinally polarized vector meson was reformulated directly in the coordinate space in the language of Operator Product Expansion (OPE) [14]. The method of Ref. [14] easily incorporates an asymmetry of the gluon distribution in the nucleon which is important for the whole problem [15]. Moreover, higher twist DA's appear quite naturally in this approach. Though this paper deals with a transversely polarized vector meson for which the method of [14 must be somewhat modified, as a by-product of our study we show that there are no three-particle DA's in the diffractive amplitude of Ref. [3, 14] ( see the end of Sect.3). Among other potential applications one can mention the light

\footnotetext{
*See Ref. 11] for a recent re-analysis of leading twist $\rho$-meson DA's.
} 
cone QCD sum rule approach [16] to semileptonic and radiative B-decays [17] and (non-)factorization in non-leptonic B-decays [18]. Besides these practical issues, our results may have some conceptual importance as they seem to imply a breakdown of OPE for some exclusive processes at the nonleading twist level. The letter is organized as follows. In Sect.2 we review the conformal group technique of Ref. [6]. Its modification for the case of interest is presented in Sect.3 where we construct a complete set of asymptotic three particle DA's for a transversely polarized massive vector meson. Sect.4 contains a comparison with previous analyses and discussion of possible consequences of our results.

\section{Conformal symmetry on the light cone and asymptotic distribution amplitudes}

The aim of this section is to remind the algebra of the so-called collinear conformal group obtained by the reduction of the full conformal group to fields varying on the light cone $x^{2}=0$ and establish a projection operator technique and general formulae for asymptotic distribution amplitudes with arbitrary number of constituents.

The algebra of the conformal group $\mathrm{O}(4,2)$ is obtained by adding the generators $D$ of dilatations and $K_{\mu}$ of special conformal transformations to the generators $P_{\mu}$ and $M_{\mu \nu}$ of the Poincare group. They form the algebra

$$
\begin{aligned}
{\left[D, K_{\mu}\right] } & =i K_{\mu},\left[D, P_{\mu}\right]=-i P_{\mu} \\
{\left[D, M_{\mu \nu}\right] } & =0,\left[K_{\mu}, K_{\nu}\right]=0 \\
{\left[K_{\mu}, P_{\nu}\right] } & =-2 i\left(g_{\mu \nu} D+M_{\mu \nu}\right) \\
{\left[K_{\alpha}, M_{\mu \nu}\right] } & =i\left(g_{\alpha \mu} K_{\nu}-g_{\alpha \nu} K_{\mu}\right)
\end{aligned}
$$

in addition to the commutation relations of the Poincare algebra. These generators act on a field $\Phi(x)$ with the spin $s$ and canonical dimension $l$ as follows

$$
\begin{aligned}
{\left[P_{\mu}, \Phi(x)\right] } & =-i \partial_{\mu} \Phi(x) \\
{\left[M_{\mu \nu}, \Phi(x)\right] } & =\left[i\left(x_{\nu} \partial_{\mu}-x_{\mu} \partial_{\nu}\right)-\Sigma_{\mu \nu}\right] \Phi(x) \\
{[D, \Phi(x)] } & =-i\left(x_{\alpha} \partial_{\alpha}+l\right) \Phi(x) \\
{\left[K_{\mu}, \Phi(x)\right] } & =-i\left(2 x_{\mu} x_{\alpha} \partial_{\alpha}-x^{2} \partial_{\mu}+2 x_{\mu} l-2 i x_{\nu} \Sigma_{\mu \nu}\right) \Phi(x)
\end{aligned}
$$

where $\Sigma_{\mu \nu}$ is the spin generator

$$
\Sigma_{\mu \nu} \Psi=\frac{1}{2} \sigma_{\mu \nu} \Psi, \Sigma_{\mu \nu} G_{\alpha \beta}=i\left(\delta_{\mu \alpha} G_{\nu \beta}-\delta_{\nu \alpha} G_{\mu \beta}\right)-(\alpha \leftrightarrow \beta)
$$

For an analysis of the light cone hadron distribution amplitudes we only need to consider the action of the conformal generators (11) on a field $\Phi(u x)$ varying on the 
light cone $x^{2}=0$. It is convenient to simplify the algebra by introducing two light like vectors $x_{\mu}, \bar{x}_{\mu}$ with $x^{2}=\bar{x}^{2}=0,(x \bar{x})=1$ and defining projections on these vectors $\gamma .=\gamma_{\mu} x_{\mu}, \gamma_{*}=\gamma_{\mu} \bar{x}_{\mu}$ etc. Then the only nontrivial transformations on the field $\Phi(u x)$ are given by the components $P ., D, M_{*}$. $K_{*}$. These generators form the algebra of the so-called collinear conformal subgroup $S O(2,1) \simeq S U(1,1) \simeq S L_{2}(R)$ of projective transformations on the line. Its algebra reads

$$
\begin{aligned}
{\left[J_{+}, J_{-}\right] } & =-2 J_{3} \quad,\left[J_{3}, J_{ \pm}\right]= \pm J_{ \pm} \\
{\left[J^{2}, J_{i}\right] } & =0,\left[E, J_{i}\right]=0
\end{aligned}
$$

where we defined the operators $J_{i}$ as linear combinations of the conformal operators

$$
\begin{aligned}
J_{+} & =J_{1}+i J_{2}=\frac{i}{\sqrt{2}} P ., J_{-}=J_{1}-i J_{2}=\frac{i}{\sqrt{2}} K_{*} \\
J_{3} & =\frac{i}{2}\left(D+M_{* .}\right), E=\frac{i}{2}\left(D-M_{*}\right) \\
J^{2} & =J_{3}^{2}-J_{1}^{2}-J_{2}^{2}=J_{3}^{2}-J_{3}-J_{+} J_{-}
\end{aligned}
$$

Here two observations are in order. First, the light like vectors $x_{\mu}, \bar{x}_{\mu}$ introduced above are quite arbitrary at this stage. However, they have to be built up from physical vectors characteristic to a meson in order to get informative constraints for distribution amplitudes. Ways to do this will be described in the next section. Second, we note that the use of the collinear conformal group requires fixing a projection of the Lorentz spin of the field $\Phi(u x)$ onto the line $x_{\mu}$

$$
\Sigma_{*} \Phi(u x)=i s \Phi(x)
$$

In this case the field $\Phi(u x)$ is an eigenstate of the Casimir operator $J^{2}$ :

$$
\left[J^{2}, \Phi(u x)\right]=j(j-1) \Phi(u x)
$$

where $j=1 / 2(l+s)$ is the conformal spin of the field $\Phi(u x)$. The fixed spin components of the quark $\Psi(u x)$ and gluon $G_{\mu \nu}(u x)$ fields can be readily constructed as follows. The quark field contains two components with the spin projections $s= \pm 1 / 2$ which can be selected by the projection operators $\gamma$. and $\gamma_{*}$ :

$$
\begin{aligned}
\Sigma_{*} \cdot \gamma \cdot \Psi & =\frac{1}{2} \sigma_{*} \cdot \gamma \cdot \Psi=+i \frac{1}{2} \gamma \cdot \Psi \\
\Sigma_{*} \cdot \gamma_{*} \Psi & =\frac{1}{2} \sigma_{*} \cdot \gamma_{*} \Psi=-i \frac{1}{2} \gamma_{*} \Psi
\end{aligned}
$$

For the gluon field the relevant components with the spin projections $s=0, \pm 1$ are the following

$$
\Sigma_{*} \cdot G_{\cdot \perp}=+i G_{\cdot \perp}, \Sigma_{*} \cdot G_{* \perp}=-i G_{* \perp}, \Sigma_{*} \cdot G_{*}=\Sigma_{* \cdot} G_{\perp \perp}=0
$$

\footnotetext{
†Note the "wrong" sign of the commutator $\left[J_{+}, J_{-}\right]$in comparison to the rotation group $\mathrm{SO}(3)$.
} 
In the last equation the label $\perp$ stands for projections on the third vector $e_{\alpha}^{(\perp)}$ which is required to satisfy $\left(\bar{x} e^{(\perp)}\right)=0$.

In an analysis of hadron DA's we deal with matrix elements of the form

$$
\left\langle 0\left|\Phi_{1}\left(u_{1} x\right) \ldots \Phi_{k}\left(u_{k} x\right)\right| h(q)\right\rangle=\int D \alpha e^{-i q x\left(u_{1} \alpha_{1}+\ldots+u_{k} \alpha_{k}\right)} \phi\left(\alpha_{1}, \ldots, \alpha_{k}\right)
$$

where $D \alpha=d \alpha_{1} \cdots \alpha_{k} \delta\left(\sum_{i} \alpha_{i}-1\right)$. Here $h$ is a hadron of interest and $\phi\left(\alpha_{1}, \ldots, \alpha_{k}\right)$ stands for a corresponding multiparticle DA. The fields $\Phi_{i}\left(u_{i} x\right)$ are arranged along the light cone $x^{2}=0$. The light cone conformal symmetry suggests the following way for the analysis of the DA $\phi\left(\alpha_{1}, \ldots, \alpha_{k}\right)$. First, we choose fixed spin components of the fields $\Phi_{i}\left(u_{i} x\right)$ according to Eqs.(8),(9). Next, we note that an expansion of the tensor product $\Phi_{1}\left(u_{1} x\right) \ldots \Phi_{k}\left(u_{k} x\right)$ into irreducible representations of the collinear conformal group induces a respective decomposition for the function $\phi\left(\alpha_{1}, \ldots, \alpha_{k}\right)$ defined on the simplex $\alpha_{1}+\ldots+\alpha_{k}=1$. Therefore the evaluation of the DA $\phi\left(\alpha_{1}, \ldots, \alpha_{k}\right)$ amounts to a calculation of corresponding Clebsch-Gordan coefficients in an expansions of a tensor product into irreducible representations with a fixed value of the total conformal spin. An asymptotic DA is then defined as a representation with the lowest conformal spin which is $j_{\min }=j_{1}+\ldots j_{k}$. A general formula for the asymptotic form of arbitrary multiparticle DA has been found in Ref. [6] exploiting the fact that the collinear conformal group (4) is identical to the Lorentz group in $(2+1)$ dimensions. It reads

$$
\phi_{a s}\left(\alpha_{1}, \ldots, \alpha_{k}\right)=\frac{\Gamma\left(2 j_{1}+\ldots+2 j_{k}\right)}{\Gamma\left(2 j_{1}\right) \ldots \Gamma\left(2 j_{k}\right)} \alpha_{1}^{2 j_{1}-1} \ldots \alpha_{k}^{2 j_{k}-1} \quad, \quad \int D \alpha \phi_{a s}=1
$$

Irreducible representations with higher spins are obtained by the multiplications of (11) by mutually orthogonal polynomials of dimensions $j-\sum_{i} j_{i}$ and correspond to conformal corrections to the asymptotic multiparticle DA $\phi\left(\alpha_{1}, \ldots, \alpha_{k}\right)$ which serves as the weight function at the space of conformal polynomials. These conformal corrections will not be discussed in the present paper.

Going over to interacting fields we insert the gauge factors $[u x, v x]=$ $P \exp \left[i g \int_{v}^{u} d t x_{\mu} A_{\mu}(t x)\right]$ in between the field operators in Eq.(10) which is equivalent to the substitution of ordinary derivatives by covariant ones in the language of local conformal operators. Then contributions of different conformal spins in DA do not mix under renormalization to the one-loop accuracy [7]. This property is sufficient to construct an expansion of the leading twist DA over a series of polynomials with multiplicatively renormalizable coefficients, as in this case all representations are nondegenerate. Moreover, anomalous dimensions are ordered with the conformal spin for the leading twist. For higher twist DA's irreducible representations with sufficiently high spins are degenerate and conformal operators do not completely diagonalize the mixing matrix which only becomes "conformal block-diagonal". Still, anomalous dimensions are typically lowest for operators of minimal conformal spin. These operators are therefore leading ones as $Q^{2} \rightarrow \infty$ and thus in this case the definition (11) is justified. 


\section{Distribution amplitudes of a massive vector me- son}

In this section we explain how the general analysis of the proceeding section works in the study of three-particle quark-antiquark-gluon DA's of twist 3 and 4 of a massive vector meson. We consider a task of taking into account kinematical corrections due to a nonvanishing meson mass while the constituents of a meson are assumed to be massless. Thus, our results apply directly to the $\rho-, \omega-, \phi-$ mesons $(\mathrm{SU}(3)$ limit is implied) and also can be used for the charmonium in situations where one can neglect the c-quark mass. For definiteness, we consider the case of the charged $\rho$-meson, while modifications to be done for other mesons are trivial.

We start with a set of three particle DA's of twist 3 and 4 defined by the matrix elements of the following non-local light cone string operators

$$
\begin{array}{r}
\left\langle 0\left|\bar{d}(-x) \gamma_{\alpha} i g G_{\mu \nu}(v x) u(x)\right| \rho(q)\right\rangle=q_{\alpha}\left(\varepsilon_{\mu}^{(\lambda)} q_{\nu}-\varepsilon_{\nu}^{(\lambda)} q_{\mu}\right) \int D\left(\alpha_{i} q x\right) \Phi^{(1)}\left(\alpha_{i}\right) \\
+\varepsilon_{\alpha}^{(\lambda)}\left(q_{\mu} x_{\nu}-q_{\nu} x_{\mu}\right) \frac{m^{2}}{q x} \int D\left(\alpha_{i} q x\right) \Phi^{(2)}\left(\alpha_{i}\right) \\
+\left(\varepsilon_{\mu}^{(\lambda)} \delta_{\alpha \nu}-\varepsilon_{\nu}^{(\lambda)} \delta_{\alpha \mu}\right) m^{2} \int D\left(\alpha_{i} q x\right) \Phi^{(3)}\left(\alpha_{i}\right) \\
\left\langle 0\left|\bar{d}(-x) \gamma_{\alpha} \gamma_{5} g \tilde{G}_{\mu \nu}(v x) u(x)\right| \rho(q)\right\rangle=q_{\alpha}\left(\varepsilon_{\mu}^{(\lambda)} q_{\nu}-\varepsilon_{\nu}^{(\lambda)} q_{\mu}\right) \int D\left(\alpha_{i} q x\right) \Psi^{(1)}\left(\alpha_{i}\right) \\
+\varepsilon_{\alpha}^{(\lambda)}\left(q_{\mu} x_{\nu}-q_{\nu} x_{\mu}\right) \frac{m^{2}}{q x} \int D\left(\alpha_{i} q x\right) \Psi^{(2)}\left(\alpha_{i}\right) \\
+\left(\varepsilon_{\mu}^{(\lambda)} \delta_{\alpha \nu}-\varepsilon_{\nu}^{(\lambda)} \delta_{\alpha \mu}\right) m^{2} \int D\left(\alpha_{i} q x\right) \Psi^{(3)}\left(\alpha_{i}\right)
\end{array}
$$

Here

$$
\int D\left(\alpha_{i} q x\right)=\int d \alpha_{1} d \alpha_{2} d \alpha_{3} \delta\left(1-\alpha_{1}-\alpha_{2}-\alpha_{3}\right) e^{-i q x\left(\alpha_{1}-\alpha_{2}+v \alpha_{3}\right)}
$$

and $m^{2}$ stands for the meson mass. One can check that the DA's $\Phi^{(1)}, \Psi^{(1)}$ corespond to operators of twist 3 , while the DA's $\Phi^{(2)}, \Phi^{(3)}, \Psi^{(2)}, \Psi^{(3)}$ contain contributions of operators of both twist 3 and 4 . This can be seen using the operator identity [9]

$$
\begin{aligned}
{\left[\bar{\psi}(-x) \gamma_{\alpha} \gamma_{5} g \tilde{G}_{\mu \cdot}(v x) \psi(x)\right]^{t w-3} } & =\int_{0}^{1} d u \frac{1+u^{2}}{2} \frac{\partial}{\partial x_{\alpha}} \bar{\psi}(-u x) \gamma \cdot \gamma_{5} g \tilde{G}_{\mu \cdot}(u v x) \psi(u x) \\
& +\int_{0}^{1} d u \frac{1-u^{2}}{2} \frac{\partial}{\partial x_{\mu}} \bar{\psi}(-u x) \gamma \cdot \gamma_{5} g \tilde{G}_{\alpha \cdot}(u v x) \psi(u x)
\end{aligned}
$$

Multiplying (15) by $x_{\alpha}$, integrating by parts and taking matrix elements in accord with (13), one arrives at identities for the DA's $\Phi^{(1)}, \Psi^{(1)}$. We further note that the $\rho$ meson in Eqs.(12), (13) can only be transversely polarized. Indeed, the total helicity

\footnotetext{
${ }^{\ddagger}$ We assume the Fock-Schwinger gauge $x_{\mu} A_{\mu}(x)=0$. In a general case path-ordered gauge factors between the fields in (12,13) are implied, as required by the gauge invariance.
} 
of the quark-antiquark pair is zero for the vector or axial channel. Therefore, in order to have a longitudinally polarized meson the gluon must also be longitudinal. This is, however, forbidden by the gauge invariance as the hadron constituents in DA are nearly on-shell. This argument has been known for some time [20]. Still, as it has a somewhat hand waving flavor (the meaning of being "nearly on-shell" is not quite clear), we have formally checked this statement by allowing for longitudinal structures in (12, 13) and respective extending a set of conformal constraints (see Eqs.(19-21) below). A corresponding system of equations turns out inconsistent, which means that the longitudinal polarization in (12,13) is not possible. It is then easy to see that the Lorentz structure displayed in Eqs.(12),(13) is the most general decomposition to the $\mathrm{O}$ (twist-5) accuracy. In the early analysis of Ref. [5] only the twist 3 structures $\Phi^{(1)}, \Psi^{(1)}$ were retained on the ground of the fact that they correspond to leading power corrections in exclusive amplitudes. They have further been analysed within the QCD sum rules technique. We are going to extent the analysis of the DA's (12,13) by a completely different method which is also able to fix the "twist 4 " BDA's $\Phi^{(2)}, \Phi^{(3)}, \Psi^{(2)}, \Psi^{(3)}$. Besides of obvious practical interest, we find that our new results for the "twist 4" DA's may have some principal importance as well. The point is that, as will be shown below, there is a very nontrivial interplay between the expansions in twist and conformal spin in the problem at hand. Nonleading "twist 4" DA's have lower in comparison to twist 3 DA's conformal spins, i.e. are given by polynomials of lower dimensions. This observation may imply a breakdown of OPE for some processes at the nonleading twist level (see Sect.5). In what follows we restrict our study to the asymptotic DA's, i.e. work to the leading order in the conformal spin expansion. A calculation of first pre-asymptotic corrections is both possible and desirable. Unfortunately, it is notoriously more difficult in comparison to the pion case [6] due to a larger number of possible Lorentz structures in matrix elements corresponding to conformal corrections.

After these lengthy remarks we proceed to the conformal analysis of the DA's (12), (13). As has been explained in the previous Sect.2, to this end we need to construct two light like vectors $x_{\mu}, \bar{x}_{\mu}$. As for the first one, it is most conveniently chosen to be just the light cone coordinate $x_{\mu}$ which enters the definitions (12), (13) (this justifies the identical notations for both). A form of the second light like vector $\bar{x}_{\mu}$ is a bit less obvious. The choice $\bar{x}_{\mu}=q_{\mu} /(q x)$ which was used in Ref. [6] for the analysis of the pion DA's clearly does not work in the case at hand as now $q^{2} \neq 0$. The correct form of the vector $\bar{x}_{\mu}$ for a massive vector meson with arbitrary polarization is given by

$$
\bar{x}_{\mu}=\frac{q_{\mu}+m \varepsilon_{\mu}^{(\lambda)}}{q x+m\left(\varepsilon^{(\lambda)} x\right)} \quad, \quad \bar{x}^{2}=0
$$

supplemented with $\left(\varepsilon^{(\lambda)} e^{(\perp)}\right)=0$. It is easy to check that with such choice the

\footnotetext{
${ }^{\S}$ Here we put the quotation marks in order to remind that these DA's give rise to further power corrections in exclusive amplitudes in comparison to those produced by the DA's $\Phi^{(1)}, \Psi^{(1)}$. One has, however, to bear in mind that in the operatorial sense they contain contributions of twist 3 , as can be seen from Eqs. (17-19).
} 
projection formulae (8),(9) and the whole analysis of Sect.2 remain valid in the massive case.

Selecting different spin components in Eq.(12) according to (8),(9),(16) we obtain the following system

$$
\begin{gathered}
\left\langle 0\left|\bar{d}(-x) \gamma . i g G_{* \cdot}(v x) u(x)\right| \rho(q)\right\rangle=-m(q x) \int D\left(\alpha_{i} q x\right) \Phi^{(1)} \\
\left\langle 0\left|\bar{d}(-x) \gamma_{*} i g G_{*} \cdot(v x) u(x)\right| \rho(q)\right\rangle=\frac{m^{3}}{(q x)} \int D\left(\alpha_{i} q x\right)\left(\Phi^{(2)}-\Phi^{(1)}-\Phi^{(3)}\right) \\
\left\langle 0\left|\bar{d}(-x) \gamma_{\perp} i g G_{\perp *}(v x) u(x)\right| \rho(q)\right\rangle=\left(e^{(\perp)} e^{(\perp)}\right) \frac{m^{3}}{(q x)} \int D\left(\alpha_{i} q x\right) \Phi^{(3)} \\
\left\langle 0\left|\bar{d}(-x) \gamma_{\perp} i g G_{\perp .}(v x) u(x)\right| \rho(q)\right\rangle=0
\end{gathered}
$$

plus analogous equations for the set (13) which are obtained from (17 - 20) by the substitution $i g G \rightarrow \gamma_{5} g \tilde{G}, \Phi^{(i)} \rightarrow \Psi^{(i)}$. We have used the fact that for the transversely polarized $\rho$-meson $\left(\varepsilon^{(\lambda)} x\right)=0$ for $x^{2}=0$. We will now study separately each of the Eqs.(17-19). Let us start with the first couple of equations. Using the general formula (11) we write

$$
\begin{aligned}
\left\langle 0\left|\bar{d}(-x) \gamma \cdot i g G_{* \cdot}(v x) u(x)\right| \rho(q)\right\rangle & =120 a_{1} \int D\left(\alpha_{i} q x\right) \alpha_{1} \alpha_{2} \alpha_{3}(1+\ldots) \\
\left\langle 0\left|\bar{d}(-x) \gamma \cdot \gamma_{5} g \tilde{G}_{* \cdot}(v x) u(x)\right| \rho(q)\right\rangle & =120 b_{1} \int D\left(\alpha_{i} q x\right) \alpha_{1} \alpha_{2} \alpha_{3}(1+\ldots)
\end{aligned}
$$

We introduce here matrix elements of local operators

$$
\begin{aligned}
\left\langle 0\left|\bar{d} \gamma_{\alpha} i g G_{\mu \nu} u\right| \rho(q)\right\rangle & =q_{\alpha}\left(\varepsilon_{\mu}^{(\lambda)} q_{\nu}-\varepsilon_{\nu}^{(\lambda)} q_{\mu}\right) A_{1}+\left(\varepsilon_{\mu}^{(\lambda)} \delta_{\alpha \nu}-\varepsilon_{\nu}^{(\lambda)} \delta_{\alpha \mu}\right) m^{2} A_{2} \\
\left\langle 0\left|\bar{d} \gamma_{\alpha} \gamma_{5} g \tilde{G}_{\mu \nu} u\right| \rho(q)\right\rangle & =q_{\alpha}\left(\varepsilon_{\mu}^{(\lambda)} q_{\nu}-\varepsilon_{\nu}^{(\lambda)} q_{\mu}\right) B_{1}+\left(\varepsilon_{\mu}^{(\lambda)} \delta_{\alpha \nu}-\varepsilon_{\nu}^{(\lambda)} \delta_{\alpha \mu}\right) m^{2} B_{2}
\end{aligned}
$$

It is clear that $A_{1}=A_{2}=0$ by the G-parity, while $B_{1}$ and $B_{2}$ are both non-zero and will be calculated below. It is then easy to express the normalization constants $a_{1}, b_{1}$ of Eq.(21) in terms of $A_{i}$ and $B_{i}$ of (22). In this way we find

$$
\begin{aligned}
& \Phi^{(1)}=0 \\
& \Psi^{(1)}=120 B_{1} \alpha_{1} \alpha_{2} \alpha_{3}
\end{aligned}
$$

A second couple of constraints is obtained analogously from Eq.(18) and its counterpart for $\Psi_{i}$. It reads

$$
\begin{aligned}
\Phi^{(1)}-\Phi^{(2)}+\Phi^{(3)} & =0 \\
\Psi^{(1)}-\Psi^{(2)}+\Psi^{(3)} & =6\left(B_{1}+B_{2}\right) \alpha_{3}
\end{aligned}
$$

The last Eq.(19) needs more care. Note that the quark spins in (19) are not fixed by the transverse gamma matrix. Using the identity

$$
\gamma_{\perp}=-\frac{1}{2}\left(\gamma \cdot \gamma_{\perp} \gamma_{*}+\gamma_{*} \gamma_{\perp} \gamma .\right)
$$

\footnotetext{
ฯHere and in what follows ellipses stand for higher conformal spin contributions.
} 
we consider two auxiliary DA's with fixed projections of the spins of quarks on the line $x_{\mu}$ :

$$
\begin{aligned}
\left\langle 0\left|\bar{d}(-x) \gamma \cdot \gamma_{\perp} \gamma_{*} i g G_{\perp *}(v x) u(x)\right| \rho(q)\right\rangle & =6 a \int D\left(\alpha_{i} q x\right) \alpha_{2}(1+\cdots) \\
\left\langle 0\left|\bar{d}(-x) \gamma_{*} \gamma_{\perp} \gamma \cdot i g G_{\perp *}(v x) u(x)\right| \rho(q)\right\rangle & =-6 a \int D\left(\alpha_{i} q x\right) \alpha_{1}(1+\cdots)
\end{aligned}
$$

The normalization factor $a$ is the same in both expressions by the G-parity. One can see that only the antisymmetric combination of gamma matrices contributes in Eq.(26). A little algebra then yields $a=B_{2} m^{3} /(q x)$. After a similar calculation for the axial channel we obtain

$$
\begin{aligned}
\Phi^{(3)} & =3 B_{2}\left(\alpha_{1}-\alpha_{2}\right) \\
\Psi^{(3)} & =3 B_{2}\left(1-\alpha_{3}\right)
\end{aligned}
$$

Using Eqs.(23, 24, 27) we finally arrive at the following expressions for the asymptotic DA's :

$$
\begin{aligned}
& \Phi^{(1)}=0 \\
& \Phi^{(2)}=\Phi^{(3)}=3 B_{2}\left(\alpha_{1}-\alpha_{2}\right) \\
& \Psi^{(1)}=120 B_{1} \alpha_{1} \alpha_{2} \alpha_{3} \\
& \Psi^{(2)}=3 B_{2}\left(1-3 \alpha_{3}\right)+6 B_{1} \alpha_{3}\left(20 \alpha_{1} \alpha_{2}-1\right) \\
& \Psi^{(3)}=3 B_{2}\left(1-\alpha_{3}\right)
\end{aligned}
$$

Note in passing that a peculiarity of restrictions imposed by the conformal invariance can be seen e.g. in the expression for $\Psi^{(2)}$ which does not correspond to any simple minded homogenious polynomial in $\alpha_{i}$. Constants $B_{1}, B_{2}$ can be calculated by the standard QCD sum rules method. In fact, their linear combination $B_{1}+3 B_{2}$ (corresponding to the convolution with $\delta_{\alpha \nu}$ in Eq. 22 )) was found a long time ago [19. In analogy with the method used in [19] we find convenient to calculate the non-diagonal correlation function

$$
T(q)=i \int d x e^{i q x}\left\langle 0\left|T\left\{\bar{d}(x) \gamma_{\alpha} \gamma_{5} g \tilde{G}_{\mu \nu} u(x) \bar{u}(0) \gamma_{\lambda} d(0)\right\}\right| 0\right\rangle
$$

The matrix element of interest appears as the lowest intermediate hadron state contribution to the imaginary part of (29). Matching this expression to a QCD calculation at $q^{2} \rightarrow-\infty$ we obtain two sum rules for parameters $B_{1}$ and $B_{2}$. The Borel transformed sum rules look as follows (a subtraction of continuum from the perturbative term is implied):

$$
\begin{aligned}
& B_{1}=\frac{1}{m_{\rho} f_{\rho}} e^{\frac{m_{\rho}^{2}}{M^{2}}}\left[-\frac{\alpha_{s}}{72 \pi^{3}} M^{4}+\frac{1}{24}\left\langle\frac{\alpha_{s}}{\pi} G^{2}\right\rangle+\frac{40}{27} \pi \alpha_{s} \frac{\langle\bar{\psi} \psi\rangle^{2}}{M^{2}}+\cdots\right] \\
& B_{2}=\frac{1}{m_{\rho} f_{\rho}} e^{\frac{m_{\rho}^{2}}{M^{2}}}\left[-\frac{\alpha_{s}}{72 \pi^{3}} M^{4}+\frac{1}{24}\left\langle\frac{\alpha_{s}}{\pi} G^{2}\right\rangle-\frac{24}{27} \pi \alpha_{s} \frac{\langle\bar{\psi} \psi\rangle^{2}}{M^{2}}+\cdots\right]
\end{aligned}
$$


Unfortunately, the first of the sum rules in (30) is not stable as the condensate terms enter there with the same signs. Therefore we use only the second of Eqs.(30) to find the number $B_{2}$ and fix the other parameter $B_{1}$ from the known value $B_{1}+3 B_{2} \simeq$ $1.85 \cdot 10^{-2} \mathrm{GeV}^{2}$ which was obtained in Ref.[19] from a similar (stable) sum rule written directly for this combination. Numerically this procedure yields

$$
B_{1} \simeq 0.8 \cdot 10^{-2} \mathrm{GeV}^{2}, B_{2} \simeq 0.35 \cdot 10^{-2} \mathrm{GeV}^{2}
$$

These numbers provide normalizations to the complete system of asymptotic DA's (28). A comparison between our results (28) and the set of DA's suggested in Ref. [5] will be carried out in the next section. Here we would like to end up the discussion of the $\rho$-meson DA's with a remark that there exists one more series of DA's generated by the matrix element

$$
\left\langle 0\left|\bar{d}(-x) \sigma_{\mu \nu} i g G_{\alpha \beta}(v x) u(x)\right| \rho(q)\right\rangle
$$

which corresponds to a longitudinally polarized vector meson 20] as there the total quark helicity is \pm 1 and the gluon helicity is $\mp 1$. These DA's cannot contribute the diffractive amplitude of [3, 14] owing to odd number of gamma matrices in a trace. This justifies the claim made in Introduction on the absence of three particle DA's in the diffractive electroproduction amplitude of Ref.[3], 14]

\section{Discussions}

Our results (28) should be confronted with the alternative set of DA's suggested in Ref.[5] :

$$
\begin{aligned}
& \Phi^{(1)}=7 ! f_{3 \rho}^{V}\left(\alpha_{1}-\alpha_{2}\right) \alpha_{1} \alpha_{2} \alpha_{3}^{2}, \int D \alpha\left(\alpha_{1}-\alpha_{2}\right) \Phi_{\|}^{(1)}=f_{3 \rho}^{V} \simeq 0.25 \cdot 10^{-2} \mathrm{GeV}^{2} \\
& \Psi^{(1)}=360 f_{3 \rho}^{A} \alpha_{1} \alpha_{2} \alpha_{3}^{2}, \int D \alpha \Psi_{\|}^{(1)}=f_{3 \rho}^{A} \simeq 0.6 \cdot 10^{-2} \mathrm{GeV}^{2}
\end{aligned}
$$

As the DA's $\Phi^{(1)}$ in (28) and (33) are incomparable (any nontrivial $\Phi^{(1)}$ is preasymptotic within a systematic expansion in the conformal spin and thus is beyond our asymptotic accuracy), we concentrate on a comparison between the DA's $\Psi^{(1)}$. First we note that the normalization parameter $B_{1}$ of the DA's $\Psi^{(1)}$ in $(28)$ is consistent within a typical for the QCD sum rules method accuracy with the normalization $f_{3 \rho}^{A}$ in (33). However, the functional forms of $\Psi^{(1)}$ in (28) and (33) are different by an extra power of $\alpha_{3}$. A source of this discrepancy is not quite clear to us. It can be due to different definitions of twist in this paper and Ref. [5]. Our $\Psi^{(1)}$ corresponds to matrix elements of twist 3 operators, while the authors of [5] have in fact singled out symmetric parts of operators without subtraction of traces, as just these quantities determine power corrections in exclusive amplitudes. Another possible and related reason is that when (as in Ref.[5]) the DA is calculated by studying a correlation 
function of currents with derivatives together with a symmetrization procedure, the imaginary part becomes a combination of contributions of different resonances in different Lorentz structures. In this case the DA calculated from the asymptotic loop may correspond to some "average" hadron (i.e. an admixture of a hypothetic exotic meson with $J^{P C}=0^{+-}[19$ is not ruled out). We also note that from the viewpoint of the conformal spin expansion the polynomial $\alpha_{1} \alpha_{2} \alpha_{3}^{2}$ could arise from the next-to-leading term $\alpha_{1} \alpha_{2} \alpha_{3}\left(1-3 \alpha_{3}\right)$ with $j=7 / 2$, while our asymptotic $\Psi^{(1)}$ has $j=3$ 兄. A coefficient in front of this conformal correction is fixed by the $\rho$ meson matrix element of the $j=7 / 2$ operator $\bar{d} \gamma \cdot \gamma_{5} g D . \tilde{G}_{*} \cdot u$. On the other hand, it can be readily seen that this matrix element is exactly zero for the transverse polarization of the $\rho$-meson. Therefore, it seems unlikely to have a multiplicatively renormalizable combination of conformal operators giving rise to the combination $\alpha_{1} \alpha_{2} \alpha_{3}^{2}$ and having an anomalous dimension lower than that of the $j=3$ operator $\bar{d} \gamma \cdot \gamma_{5} g \tilde{G}_{*} . u$ which corresponds to the polynomial $\alpha_{1} \alpha_{2} \alpha_{3}$. To summarize, we see a few possible explanations for the difference by a power of $\alpha_{3}$ between the DA's $\Psi^{(1)}$ in (28) and (33), but for its precise identification a further analysis is needed. While the choice (33) may be ultimately true for the pQCD method of Ref. [5], it seems that it is the DA $\Psi^{(1)}$ of Eq. (28) that has to be used in the light cone QCD approach to "soft" exclusive processes [2], since there one deals directly with the matrix elements (12,13).

Now we would like to discuss the non-leading "twist 4" DA's $\Phi^{(2)}, \Phi^{(3)}, \Psi^{(2)}, \Psi^{(3)}$ in (28) which hitherto have not been considered in the literature. Somewhat surprisingly, we have found that though these DA's correspond to further power corrections in comparison to those produced by the twist 3 DA's $\Phi^{(1)}, \Psi^{(1)}$, their conformal spins are lower $\left(j=2\right.$ for $\Phi^{(2)}, \Phi^{(3)}$, and $\Psi^{(3)}$, while $\Psi^{(2)}$ is a combination of $j=2$ and $j=3$ pieces). Respectively, they are given by polynomials of lower dimensions than the asymptotic DA $\Psi^{(1)}$ in $(28)$. On the other hand, we remind that typically DA's enter exclusive amplitudes through some integrals like $\int \Phi\left(\alpha_{i}\right) / \alpha_{3}^{2}$, cf. Ref. [5]. Powers of $\alpha_{i}$ in the denominator originate from quark and gluon propagators and are therefore the same in terms corresponding to three particle DA's of twist 3 and 4. Moreover, in exclusive amplitudes including three particle DA's of the $\rho$-meson calculated in [5] corresponding integrals contain logarithmic end-point divergences. Our result (28) then implies that if no special cancellations occur (we see no reason why they should), in such a situation an attempt to evaluate the twist 4 contribution will give rise to power infrared divergences ${ }^{* *}$. This would mean a breakdown of OPE for this process and invalidation of a calculated twist 3 contribution. This observation can be of wider sense and imply that OPE may be inapplicable in exclu-

\footnotetext{
${ }^{\|}$A first possible non-zero term in the DA $\Phi^{(1)}$ corresponds to the polynomial $\left(\alpha_{1}-\alpha_{2}\right) \alpha_{1} \alpha_{2} \alpha_{3}$ with $j=7 / 2$. The same power of $\alpha_{3}$ seems to be missing there as well.

**Another possibility (V. Braun, private communication) is that power infrared divergences may cancel by virtue of equations of motion so that only logarithmic end-point singularities will survive. This scenario seems unlikely as the DA's (28) are not related by the equations of motion. Dynamical calculations are needed to see whether cancellations of infrared divergences take place in physical amplitudes.
} 
sive reactions beyond the leading twist order. This conjecture does not contradict to anything we currently understand about exclusive processes. To illustrate this point, we consider two examples.

1. In the most well studied case of the pion electromagnetic form factor the short distance regime breaks down at the $1 / Q^{4}$ order as there the soft Feynman-type mechanism [2] brings in the same $1 / Q^{4}$ contribution as a twist 4 part of the hard rescattering amplitude (we leave aside the mild Sudakov suppression in the former). 2. For the $\gamma \rho \pi$ transition form factor, which is a nonleading twist process [5] related to the helicity flipping, the hard rescattering diagram yields the asymptotic $1 / Q^{4}$ behavior. However, the soft Feynman contribution gives the same $1 / Q^{4}$ [2], and therefore the short distance regime in this process breaks down already at the leading order in $1 / Q^{2}$. If the above scenario were to occur, it would just signal on this breakdown, which is otherwise not seen in the leading hard rescattering amplitude taken on its own (logarithmic end-point divergences are relatively harmless as they can be cured by the Sudakov correction factor).

A common feature in these two examples is that in the perturbative QCD approach the short distance regime is implied within the hard gluon exchange mechanism. This assumption certainly does not hold at the nonleading twist level as the $1 / Q^{4}$ parts of the hard amplitudes are just the radiative corrections to the soft Feynmantype contributions which have nothing to do with short distances. Thus it is not unreasonable to expect divergences at the next order of OPE for these processes as a manifestation of the same fact. It would be challenging to see whether there exist physical motivations to expect a breakdown of OPE in other and, in particular, higher twist exclusive processes like $\psi \rightarrow \rho \pi$ which seems suggestive according to formulae given in Ref. [5]. To avoid possible misunderstanding, we should stress that in the light cone QCD sum rule approach to exclusive processes [2] the situation is different as there the light cone dominance is enforced from outside by an analytic continuation to the deep Euclidean region. Therefore, we do not expect a similar pattern of a breakdown of OPE in this case, though this possibility cannot be ruled out.

\section{Acknowledgments}

I am grateful to A. Zhitnitsky for stimulating discussions. Thanks are due to V. Braun for reading the manuscript and useful comments. 


\section{References}

[1] S.J. Brodsky and G.P. Lepage, in "Perturbative Quantum Chromodynamics" edited by A.H. Mueller, World Scientific Publishing Co.,1989.

[2] V. Braun and I. Halperin, Phys. Lett. B328 (1994) 457.

[3] S.J. Brodsky, L.L. Frankfurt, J.F. Gunion, A.H. Mueller and M. Strikman, Phys. Rev. D50 (1994) 3134.

[4] V.M. Belyaev and M.B. Johnson, hep-ph/9605279, hep-ph/9702207.

[5] V.L. Chernyak and A.R. Zhitnitsky, Phys.Rep. 112 (1984) 173.

A.R. Zhitnitsky, I.R. Zhitnitsky and V.L. Chernyak, Sov. J. Nucl. Phys. 38 (1983) 645,775; 41 (1985) 284.

[6] V.M. Braun and I.E. Filyanov, Z.Phys. C48 (1990) 239.

[7] Yu.M. Makeenko, Yad. Fiz. 33 (1981) 842.

[8] Th. Ohrndorf, Nucl. Phys. B198 (1982) 26.

[9] I.I. Balitsky and V.M. Braun, Nucl. Phys. B311 (1988/1989) 541.

[10] S.J. Brodsky and G.P. Lepage, Phys. Lett. B87 (1979) 359.

A.V. Efremov and A.V. Radyushkin, Phys. Lett. B94 (1980) 245.

[11] P. Ball and V.M. Braun, Phys. Rev. D54 (1996) 2182.

[12] M. Derrick et al., Phys. Lett. B356 (1995) 601.

M. Arneodo et al., Nucl. Phys. B429 (1994) 503.

S. Aid et al., Nucl. Phys.B468 (1996) 3.

[13] L. Frankfurt, W. Koepf and M. Strikman, hep-ph/9702216.

[14] I. Halperin and A. Zhitnitsky, hep-ph/9612425.

[15] A.V. Radyushkin, Phys. Lett. B385 (1996) 333 ; hep-ph/9704207.

[16] V.M. Braun and I.E. Filyanov, Z.Phys. C44 (1989) 157.

[17] A. Ali, V.M. Braun and H. Simma, Z.Phys. C63 (1994) 437.

[18] I. Halperin, Phys. Lett. B349 (1995) 548.

[19] V.M. Braun and A.V. Koleshichenko, Phys. Lett. 175B (1986) 485.

[20] M. Benayoun, V.L. Chernyak and I.R. Zhitnitsky, Nucl. Phys. B348 (1991) 327. 